\title{
A GLIMPSE ON BIRKHOFF-JAMES ORTHOGONALITY IN BANACH SPACES
}

\author{
B. P. OJHA AND P. M. BAJRACHARYA
}

\begin{abstract}
This paper is an overview of various results on Birkhoff-James orthogonality of operators in Hilbert space and Banach spaces. We mainly focus on Birkhoff orthogonality of linear(bounded and compact) operators in terms of matrices, projection angles, Hilbert $C^{*}$-modules as well as on Banach modules. The article concludes with some open problems regarding possible correlation between Birkhoff-James orthogonality and Carlsson orthogonality, particularly in the case of Pythagorean orthogonality.

Дано огляд різноманітних результатів щодо ортогональності в сенсі БіркгофаДжеймса операторів у гільбертових і банахових просторах. Переважно розглядається ортогональність за Біркгофом лінійних (обмежених і компактних) операторів у термінах матриць, кутів, гільбертових $\mathrm{C}^{*}$-модулів, а також банахових модулів. Наведені деякі відкриті питання стосовно співвідношень ортогональністю Біркгофа-Джеймса та ортогональністю Карлссона, зокрема для випадку піфагорової ортогональності.
\end{abstract}

\section{INTRODUCTION}

The concept of Birkhoff orthogonality began in 1935 [1. In the literature of orthogonality this is known with some other names such as; Birkhoff- James orthogonality and Blaschke Birkhoff-James orthogonality ( see 2]). In this paper [1, 3], an orthogonality which satisfies homogeneity but neither symmetric nor additive is defined by $x \perp y$ if and only if $\|x+\lambda y\| \geq\|x\|$ for all $\lambda$, is known as Birkhoff orthogonality or Birkhoff-James orthogonality. The geometrical meaning of Birkhoff orthogonality is that if $\mathrm{x}$ is an unit vector of a Banach space $\mathrm{X}$ and $y \in X$, then $\mathrm{x}$ is Birkhoff orthogonal to y means that the straight line $\{x+\lambda y: \lambda \in K\}$ is tangent to the unit ball of $\mathrm{X}$ at $\mathrm{x}$. This concept is similar to the statement: suppose two lines $l_{1}$ and $l_{2}$ intersect at the point $\mathrm{m}$, then $l_{1} \perp l_{2}$ if and only if the distance from a point of $l_{2}$ to a given point $\mathrm{n}$ of $l_{1}$ is never less than the distance from $\mathrm{m}$ and $\mathrm{n}$. [3] For any hyper-plane $H \subset X$, $\mathrm{x}$ is said to be orthogonal to $\mathrm{H}$ if $\forall x \in H, x \perp h$.

Bhatia and Semrl in [4 generalize the definition of Birkhoff orthogonality in terms of matrices. For any matrices $\mathrm{A}$ and $\mathrm{B}$ they denote the symbol $\|A\|$ for operator norm of $\mathrm{A}$ and $\mathrm{A}$ is orthogonal to $\mathrm{B}$ in the sense of Birkhoff-James iff for any complex number z, $\|A+z B\| \geq\|A\|$. A matrix $\mathrm{A}$ is orthogonal to $\mathrm{B}$ iff there exist a unit vector $x \in H$ such that $\|A x\|=\|A\|$ and $\langle A x, B x\rangle=0$ [4]. They also introduced Birkhoff- James orthogonality in [4] as $A \perp B$ if and only if $\|A+z B\|_{p} \geq\|A\|_{p}$, where $\|A\|_{p}$ denotes Schatten p-norm of A defined by $\|A\|_{p}=\left[\sum_{j=1}^{n} S_{j}(A)^{p}\right]^{\frac{1}{p}}$ for $1 \leq p<\infty$ and $S_{1}(A) \geq$ ...... $S_{n}(A)$ are singular values of A. Taking the special case for $p=2$, Bhatia and Semrl in [4] also proved that the given orthogonality is equivalent to usual Hilbert space condition $\langle A, B\rangle=0$, which defines an inner-product on the space of matrices as $\langle A, B\rangle=\operatorname{tr}\left(A^{*} B\right)$. The norm associated to this inner product is $\|\cdot\|_{2}$. In an infinite dimensional case [4, for

2020 Mathematics Subject Classification. 45E15, 46J10.

Keywords. Birkhoff-James orthogonality, Banach modules, Carlsson orthogonality, Robert orthogonality. 
any bounded operators in a Hilbert space $\mathrm{H}, A \perp B$ if and only if there exist a sequence $\left\{x_{n}\right\}$ of unit vectors such in $\mathrm{H}$ that $\|A x\| \rightarrow\|A\|$, and $\left\langle A x_{n}, B x_{n}\right\rangle \rightarrow 0$.

Benitz et al. [5] proved that $\mathrm{X}$ is an inner-product space if and only if for any linear operators $\mathrm{A}$ and $\mathrm{C}$ in a finite dimensional normed space $\mathrm{X}, A \perp C \Leftrightarrow \exists u \in S_{X}:\|A u\|=$ $\|u\|, A u \perp C u$, where $S_{X}=\{x \in X:\|x\|=1\}$ and " $\perp$ " denotes the Birkhoff-James orthogonality.

Theorem 1.1. [5] If $S_{X}$ is not an ellipse( $X$ is not an inner-product space), then there exists linear operators $A$ and $C$ in $X$ such that $A \perp C$, but there does not exists $u \in S_{X}$ such that $\|A\|=\|A U\|$ and $A u \perp C u$.

Theorem $1.2([5])$. A real finite dimensional normed space $X$ is an inner-product space if and only if , for $A, C \in L(X), A \perp C \Leftrightarrow \exists x \in S_{X}:\|A\|=\|A x\|, A x \perp C x$.

where, $P_{x y}=D_{x y}\left(\begin{array}{ll}1 & 0 \\ 0 & 0\end{array}\right)$ and $D_{x y}=\left(\begin{array}{ll}x_{1} & y_{1} \\ x_{2} & y_{2}\end{array}\right)$.

Theorem 1.3. [6] The q-angle has the following properties:

(i) Part of parallelism property: $A_{q}(x, y)=0$ iff $x$ and $y$ are linearly dependent.

(ii) Part of homogeneity property: $A_{q}(A x, B y)=A_{q}(x, y)$ for every $x, y \in X$ and $A, B \in \mathbb{R}-\{0\}$.

In 66 Chen Zhi-Zhi et al. have given slightly different definition of Birkhoff orthogonality in such a way that; $\mathrm{x}$ is Birkhoff orthogonal to $\mathrm{y}$ iff $A_{q}(x, y)=\frac{\pi}{2}$ by using projections of the angles between two vectors $\mathrm{x}$ and $\mathrm{y}$ in a real two dimensional normed space $\mathrm{X}$.

Definition 1.4. 6] The g-angle between two vectors $\mathrm{x}$ and $\mathrm{y}$ is given by $g(x, y)=$ $\cos ^{-1} \frac{g(x, y)}{\|x\|\|y\|}$, where $g(x, y)=\frac{1}{2}\|x\|\left[\tau_{+}(x, y)+\tau_{-}(x, y)\right]$ and $\tau_{ \pm}(x, y)=\lim _{t \rightarrow \pm 0} \frac{\|x+t y\|-\|x\|}{t}$. In that case $x \perp_{g} y$ if $g(x, y)=0$ or $A_{g}(x, y)=\frac{\pi}{2}$.

For any $x=\left(x_{1}, x_{2}\right)^{T}$ and $y=\left(y_{1}, y_{2}\right)^{T}$ in a two dimensional real normed space $\mathrm{X}$,

$$
q(x, y)=\left\{\begin{array}{l}
0 \quad \text { if } \mathrm{x} \text { and } \mathrm{y} \text { are linearly dependent } \\
\left\|P_{x y}\right\|^{-1}, \text { if } \mathrm{x} \text { and } \mathrm{y} \text { are linearly independent. }
\end{array}\right.
$$

Continuity property: If $x_{n} \rightarrow x$ and $y_{n} \rightarrow y$, then $A_{q}\left(x_{n}, y_{n}\right) \rightarrow A_{q}(x, y)$, where $A_{q}(x, y)$ is q-angle between $\mathrm{x}$ and y defined by $A_{q}(x, y)=\sin ^{-1}[q(x, y)]$.

Lemma 1.5. 6] If $x$ is Birkhoff orthogonal to $y$. Then for any $m, n \in \mathbb{R},\|m x+n y\| \geq$ $\|m x\|$.

Proof. If $m=0$, the conclusion is obviously true. If $m \neq 0$ and if $\mathrm{x}$ is Birkhoff orthogonal to $\mathrm{y}$,

$$
\|m x+n y\|=|m|\left\|x+\frac{m}{n} y\right\| \geq|m|\|x\|=\|m x\| .
$$

Theorem 1.6. 7] Let $x=\left(x_{1}, x_{2}\right)^{T}$ and $y=\left(y_{1}, y_{2}\right)^{T}$ be two vectors in a two dimensional real normed space $X$ with basis $\left\{e_{1}, e_{2}\right\}$. Then $x$ is Birkhoff-orthogonal to to $y$ iff $A_{q}(x, y)=\frac{\pi}{2}$ i.e. $\left\|P_{x y}\right\|=1$.

\section{Orthogonality on $C^{*}$-Module}

[8] Let $\mathrm{A}$ be a $C^{*}$-algebra and $\mathrm{H}$ be a (left) $\mathscr{A}$ module. Suppose that the linear structure given on $\mathscr{A}$ and $\mathrm{H}$ are compatible, that is, $\lambda(a x)=a(\lambda x)$ for every $\lambda \in \mathbb{C}$ and $a \in H$. Then there exists a mapping $\langle.,\rangle:. H \times H \rightarrow \mathscr{A}$ with the following properties:

(i) $\langle x, x\rangle \geq 0$ for every $x \in H$,

(ii) $\langle x, x\rangle=0$ iff $x=0$,

(iii) $\langle x, y\rangle=\langle y, x\rangle^{*}$ for every $x, y \in H$, 
(iv) $\langle a x, y\rangle=a\langle x, y\rangle$ of every $a \in \mathscr{A}$ and $x, y \in H$,

(v) $\langle x+y, z\rangle=\langle x, z\rangle+\langle y, z\rangle$ for every $x, y, z \in H$

The pair $\{H,\langle.,\rangle$.$\} is called a (left) pre-Hilbert \mathscr{A}$ module. The map $\langle.,$.$\rangle is called an \mathscr{A}-$ valued inner-product. If the pre-Hilbert $\mathscr{A}$-module $\{H,\langle.,\rangle$.$\} is complete with respect to$ the norm $\|x\|=\|\langle x, x\rangle\|^{\frac{1}{2}}$, then it is called $\mathscr{A}$ Hilbert $C^{*}$-module over $\mathscr{A}$. Rajic et al., in [7, 8) introduced a new concept of Birkhoff-James orthogonality in a Hilbert $C^{*}$-modules over a $C^{*}$-algebra $\mathscr{A}$ and proved that such orthogonality with respect to $\mathscr{A}$-valued inner product coincide if and only if $\mathscr{A}$ is isomorphic to $\mathbb{C}$.

8 A mapping $T: V \rightarrow W$ between $\mathscr{A}$-modules $\mathrm{V}$ and $\mathrm{W}$ is called adjointable if there exists mapping $T^{*}: W \rightarrow V$ such that $\langle T x, y\rangle=\left\langle x, T^{*} y\right\rangle$ for all $v \in V, y \in W$. Such a mapping $\mathrm{T}$ is bounded, linear and satisfies $T(x a)=T(x) a$ for all $x \in V$ and $a \in$ $\mathscr{A}$. The space of all adjointable mapping from $\mathrm{V}$ into $\mathrm{W}$ is denoted by $B(V, W)$. Let $\theta_{x, y}(z)=x(y, z)$, where $\theta_{x, y} \in B(V, W)$ and $K(B, V)$ denotes the closed linear subspace of $B(V, W)$ spanned by $\left\{Q_{x y}: x \in W, y \in V\right\}$ is called space of compact operators.

Proposition 2.1. [8] Let $A, B \in B(H)$. Then $\min _{\lambda \in \mathbb{C}}\|A+\lambda B\|^{2}=\sup _{\|x i\|=1} M_{A, B}(\xi)$, where

$$
M_{A, B}(\xi)=\left\{\begin{array}{lll}
\|A \xi\|^{2}-\frac{|\langle A \xi, B \xi\rangle|^{2}}{\|B \xi\|^{2}} \text { if } & B \xi \neq 0 \\
\|A \xi\|^{2} & \text { if, } & B \xi=0
\end{array}\right.
$$

Proposition 2.2. [8] let $\mathscr{A}$ be a $C^{*}$-algebra, and $a, b \in \mathscr{A}$. Then $\min _{\lambda \in \mathbb{C}}\|a+\lambda b\|^{2}=$ $\max _{\varphi \in S(A)} M_{A, B}(\varphi)$, where

$$
M_{a, b}(\varphi)=\left\{\begin{array}{lll}
\varphi\left(a^{*} a\right)-\frac{\mid \varphi\left(a^{*} b\right)^{2}}{\varphi\left(b^{*} b\right)} & \text { if, } & \varphi\left(b^{*} b\right) \neq 0 \\
\varphi\left(a^{*} a\right) & \text { if, } & \varphi\left(b^{*} b\right)=0
\end{array}\right.
$$

Theorem 2.3. 8] Le $V$ be a Hilbert $C^{*}$-module over a $C^{*}$-algebra $\mathscr{A}$ and $x, y \in V$. Then $\min _{\varphi \in \mathbb{C}}\|x+\varphi y\|^{2}=\max _{\varphi \in S(A)} M_{x, y}(\varphi)$, where $M_{x, y}(\varphi) \in \mathscr{A}$ is defined by

$$
M_{x, y}(\varphi)=\left\{\begin{array}{lll}
\varphi(\langle x, x\rangle)-\frac{\mid \varphi(\langle x, y\rangle)^{2}}{\varphi(\langle y, y\rangle)} & \text { if, } & \varphi(\langle y, y\rangle) \neq 0 \\
\varphi(\langle x, x\rangle) & \text { if, } & \varphi(\langle y, y\rangle)=0
\end{array}\right.
$$

Theorem 2.4. [8] Let $V$ be a Hilbert $C^{*}$-module over a $C^{*}$-algebra $\mathscr{A}$. Let $x, y \in V$. Then $x \perp_{B} y \Leftrightarrow \exists \varphi \in S(\mathscr{A}): \varphi(\langle x, x\rangle)=\|x\|^{2}$ and $\varphi(\langle x, y\rangle)=0$.

Theorem 2.5. 8] Let $V$ be a Hilbert $C^{*}$-module over a $C^{*}$-algebra $\mathscr{A}$ and $x, y \in V$. Then

(i) $x \perp_{B} y \Leftrightarrow\langle x, x\rangle \perp\langle x, y\rangle \Leftrightarrow\langle x, x\rangle \perp_{B}\langle y, x\rangle$.

(ii) $x \perp_{B} y \Rightarrow x \perp_{B} x\langle x, y\rangle$ and $x \perp_{B} x\langle y, x\rangle$.

Arambasic and Rajic (see in[8]) characterized Hilbert $C^{*}$-modules where the Birkhoff orthogonality coincides with the usual orthogonality with respect to inner-product space. By using the Gelfand-Mazur theorem, it can be proved that $\mathscr{A}$ is isomorphic to $\mathbb{C}$ and using this concept, $\mathbb{C}$ is only the unital $C^{*}$-algebra in which Birkhoff orthogonality $x \perp_{B} y$ coincides with $x^{*} y=0$ for all elements $x, y \in \mathscr{A}$.

Theorem 2.6. let $V \neq\{0\}$ be a full Hilbert $\mathscr{A}$-module. then the following statements are equivalent:

(i) For all $x, y \in V$ the condition $\left(x \perp_{B} y \Leftrightarrow\langle x, y\rangle=0\right)$ is always true.

(ii) $\mathscr{A}$ is isomorphic to $\mathbb{C}$. 


\section{Generalization of Bhatia-Semrl Property}

In 2013, Sain and Paul [9] linked the Bhatia-Semrl property with norm attaining operators in a finite dimensional normed spaces which attain its norm on connected closed subset of $S_{X}$ and proved that the linear operator $\mathrm{T}$ satisfies the condition; $T \perp_{B} A \Rightarrow$ $\exists x \in D: T x \perp_{B} A x$, where $\mathrm{A}$ is a linear operator on $L(X)$ and $\mathrm{D}$ is connected closed subset of $S_{X}$. For the normed linear space X of dimension 2, their next research in 2015 (see [10]) explore the converse of previous result as obtained in [9]. They proved that if a linear operator $\mathrm{T}$ satisfies Bhatia-Semrl property, then the set of unit vectors $S_{X}$, on which $\mathrm{T}$ attains norm, is connected in the projective space $R P^{\prime}=S_{X} \backslash\{x \sim-x\}$ and conversely. For a strictly convex normed space $\mathrm{X}$, the set of operators in $L(X)$ satisfying the Bhatia-Semrl property is dense in $L(X)$. [10] Let $\mathrm{T}$ be a linear operator on a normed space $\mathrm{X}$. Then the set of unit vectors in $S_{X}$ at which $\mathrm{T}$ attains norm is given by $M_{T}=\left\{x \in S_{X}:\|T x\|=\|T\|\right\}$. Such a $\mathrm{T}$ satisfies Bhatia-Semrl property if for any operator $A \in L(X), T \perp_{B} A \Rightarrow \exists x \in M_{T}: T x \perp_{B} A x$. Sain et al. proved a slight different concept depending on the nature of $M_{T}$ described in [9] by stating that ; if $M_{T} \neq D \cup(-D)$ and the condition on the form of $M_{T}$ implying that T may not satisfies the Bhatia-Semrl property.

Theorem 3.1. 10 Let $T$ be a linear operator on a finite dimensional real normed space $X$ and $M_{T}=\left\{x \in S_{X}:\|T x\|=\|T\|\right\}$. If $M_{T}$ can be partitioned into tow non-empty sets which are contained in complementary subset of $X$, then there is a linear operator $A$ on $X$ such that $T \perp_{B} A$ but $T x \not \perp_{B} A x$.

Theorem 3.2. 10] Let $T$ be a linear operator on a finite dimensional real smooth normed space $X$. If $M_{T}=\left\{x \in S_{X}:\|T x\|=\|T\|\right\}$ is a countable set with more than 2 points. Then for any $x \in M_{T}$ there is a linear operator $A$ on $X$ such that $T \perp_{B} A$ but $T x \not_{B} A x$

Theorem 3.3. Let $T$ be a linear operator on a two dimensional real normed space $X$, and let $M_{T}=\left\{x \in S_{X}:\|T x\|=\|T\|\right\}$. If $M_{T}$ has more than two components, then for any $x \in M_{T}$ there is a linear operator $A$ on $X$ such that $T \perp_{B} A$ but $T x \not \perp_{B} A x$.

\section{Strong Birkhoff-James orthogonality}

Paul et al. in the paper 11 proved that a normed linear space $\mathrm{X}$ is strictly convex if and only if for all $x \in S_{X}$ there is bounded linear operator A which attain its norm only at the points of the form $\lambda x$ with $\lambda \in S_{k}$. To prove this, they have introduced a concept of strong Birkhoff-James orthogonality. [11] For any normed linear space X, x is said to be strongly orthogonal to $\mathrm{y}$ in the sense of Birkhoff-James iff $\|x\|<\|x+\lambda y\|$ for all $\lambda \neq 0$. The notation $x \perp S B y$ was used to indicate the strongly Birkhoff-James orthogonality and proved that the strongly Birkhoff-James orthogonality implies Birkhoff orthogonality, but the converse may not be true. To illustrate this concept, two elements $(1,0)$ and $(0,1)$ are taken in $l_{\infty}\left(\mathbb{R}^{2}\right)$, showing that $(1,0)$ and $(0,1)$ are orthogonal in the sense of Birkhoff-James but not strongly orthogonal to each other.

Definition 4.1. (Strongly orthogonal set)[11]: A finite set of elements $\left\{x_{1}, \ldots \ldots x_{k}\right\}$ is said to be strongly orthogonal set in the sense of Birkhoff-James iff for each $m \in$ $\{1,2, \ldots \ldots k\} \quad\left\|x_{m}\right\|<\left\|x_{m}+\sum_{m=1, m \neq n}^{k} \lambda_{n} x_{n}\right\|$, whenever $\lambda_{n} \neq 0$.

In case of an infinite set, if every finite subset of the set is strongly orthogonal in the sense of Birkhoff-James, then the infinite set is said to strongly orthogonal and conversely.

Theorem 4.2. [11] Let $X$ be a normed linear space and $x_{0} \in S_{x}$. If there exists a Hamel basis of $X$ containing $x_{0}$ which is strongly orthogonal relative to $x_{0}$ in the sense of Birkhoff-James, then $x_{0}$ is an extreme point of $B_{X}$. 
Theorem 4.3. 11 Let $X$ be a normed linear space and $x_{0} \in S_{X}$ be an exposed point of $B_{X}$. Then there exists a Hamel basis of $X$ containing $x_{0}$ which is strongly orthogonal relative to $x_{0}$ in the sense of Birkhoff-James.

Theorem 4.4. [11] Let $X$ be a normed linear space and $x_{0} \in S_{X}$. If there exist a Hamel basis of $X$ containing $x_{0}$ which is strongly orthogonal relative to $x_{0}$ in the sense of Birkhoff-James, then there exists a bounded invertivle linear operator $A$ on $X$ such that $\|A\|=\left\|A_{0}\right\|>\|A y\|$ for all $y \in S_{X}$ with $y \neq \lambda x_{0}, \lambda \in S_{k}$.

Theorem 4.5. [11] For a normed space $X$, and a point $x \in \operatorname{span}(X)$, the following are equivalent:

(i) $x$ is an exposed point of $B_{X}$.

(ii) There is a Hamel basis of $X$ containing $x$ which is strongly orthonormal relative to $x$ in the sense of Birkhoff-James.

(iii) There exists a bounded linear operator $A$ on $X$ which attains only at the points of the form $\lambda x$ with $\lambda \in S_{k}$.

Theorem 4.6. [11] For a normed linear space $X$, the following are equivalent.

(i) $X$ is strictly convex.

(ii) For each $x \in S_{X}$, there exist a Hamel basis of $X$ containing $x$ which is strongly orthonormal relative to $x$ in the sense of Birkhoff-James.

\section{Orthogonality of operators in complex Banach Spaces}

To study the difference of orthogonality in the complex case in comparison to the real case, Paul et al. in 2018 [12] came with a new concept of Birkhoff-James orthogonality by introducing new definitions on a complex reflexive Banach spaces and introduced more than one equivalent characterization of Birkhoff-James orthogonality of compact linear operators in the complex case. [12] For any bounded linear operator $T, A \in L(X)$, T is said to be Birkhoff-James orthogonal to $\mathrm{A}$ if $\|T+\lambda A\| \geq\|T\|$ for all $\lambda \in \mathbb{C}$ and $M_{T}=\left\{x \in S_{X}:\|T x\|=\|T\|\right\}$. In the real Banach space X, Sain introduced two sets $x^{+}$ and $x^{-}$in his paper [13] by

(i) $x^{+}=\{y \in X:\|x+\lambda y\| \geq\|x\|$ for all $\lambda \geq 0\}$ and

(ii) $x^{+}=\{y \in X:\|x+\lambda y\| \geq\|x\|$ for all $\lambda \leq 0\}$

For the complex Banach space, Paul et al. in 2018 introduced the following notations [12] depending on Sain's concept : For any $\gamma \in V$,

(i) $x_{\gamma}^{+}=\{y \in X:\|x+\lambda y\| \geq\|x\|$ for all $\lambda=t r, t \geq 0\}$

(ii) $x_{\gamma}^{-}=\{y \in X:\|x+\lambda y\| \geq \| x \mid$ for all $\lambda=t r, t \leq 0\}$

(iii) $x^{\frac{1}{\gamma}}=\{y \in X:\|x+\lambda y\| \geq\|x\|$ for all $\lambda=t r, t \in \mathbb{R}\}$

(iv) where $V=\{\gamma \in \mathbb{C}:|\gamma|=1, \arg (\gamma) \in[0,2 \pi]\}$.

(v) If $\mu=e^{i \pi} \gamma$, then $x_{\mu}^{+}=x_{\gamma}^{-}, x_{\mu}^{-}=x_{\gamma}^{+}$and $x^{\frac{1}{\mu}}=x^{\frac{1}{\gamma}}$. In the complex Banach space,

(vi) $x^{+}=\cap\left\{x_{\gamma}^{+}: \gamma \in V\right\}, x^{-}=\cap\left\{x_{\gamma}^{-}: \gamma \in V\right\}$ and $x^{\perp}=\cap\left\{x^{\frac{1}{\gamma}}: \gamma \in V\right\}$

Proposition 5.1. 13] Let $x, y \in X$, where $X$ is an complex Banach space and $\gamma \in V$. Then following statements are true

(i) Either $y \in x_{\gamma}^{+}$or $y \in x_{\gamma}^{-}$.

(ii) $x \perp \gamma y \Leftrightarrow y \in x_{\gamma}^{+}$or $y \in x_{\gamma}^{-}$.

(iii) $y \in x_{\gamma}^{+} \Rightarrow \eta y \in(\xi x)_{\gamma}^{+}$for all $\eta, \xi>0$.

(iv) $y \in x_{\gamma}^{+} \Rightarrow-y \in x_{\gamma}^{-}$and $y \in(-x)_{\gamma}^{-}$.

(v) $y \in x_{\gamma}^{-} \Rightarrow \eta y \in(\xi x)_{\gamma}^{-}$for all $\eta, \xi>0$.

(vi) $y \in x_{\gamma}^{-} \Rightarrow-y \in x_{\gamma}^{+}$and $y \in(-x)_{\gamma}^{+}$.

(vii) $y \in x_{\gamma}^{+} \Rightarrow \mu y \in(\mu x)_{\gamma}^{+}$for all $\mu \in \mathbb{C}$. 
(viii) $y \in x_{\gamma}^{-} \Rightarrow \mu y \in(\mu x)_{\gamma}^{-}$for all $\mu \in \mathbb{C}$.

Proposition 5.2. [13] Let $x, y \in X$, where $X$ is a complex Banach space. Then the following are true

(i) $x \perp_{B} y \Leftrightarrow y \in x^{+}$and $y \in x^{-}$.

(ii) $y \in x^{+} \Rightarrow \eta y(\xi x)^{+}$for all $\eta, \xi>0$.

(iii) $y \in x^{+} \Rightarrow-y \in x^{-}$and $y \in(-x)^{-}$.

(iv) $y \in x^{-} \Rightarrow-y \in x^{+}$and $y \in(-x)^{+}$.

(v) $y \in x^{-} \Rightarrow \eta y \in(\xi x)^{-}$for all $\eta, \xi>0$.

Theorem 5.3. 13] Let $X$ be a reflexive complex Banach space, and $Y$ be any complex Banach space. Let $T, A \in K(x, y)$. Then $T \perp_{B} A \Leftrightarrow \forall \gamma \in V, \quad \exists \quad x=x(\gamma), y=y(\gamma) \in$ $M_{T}: A x \in(T x)_{\gamma}^{+}$and $T y \in(T y)_{\gamma}^{-}$.

Theorem 5.4. 13] Let $X$ be a complex Banach Space. Let $x, y \in X$ and $r=e^{i \theta}$, where $\theta \in[0,2 \pi]$. If $y \in x_{\gamma}^{+}$, then either $y \in x_{\mu}^{+}$for all $\mu$ with $\arg \mu \in[0, \theta]$ or $y \in x_{\mu}^{+}$for all $\mu$ with $\arg \mu \in[0, \pi]$.

Theorem 5.5. [13] Let be a linear operator on a finite dimensional complex Banach space $X$, such that $M_{T}$ is a closed connected subset of $S_{X}$. Then for $A \in L(X), T \perp_{B}$ $A \Leftrightarrow \forall \gamma \in V \quad \exists \quad x=x(\gamma) \in M_{T}: T x \perp_{\aleph} A x$.

Theorem 5.6. [13] Let $T$ be a linear operator in a finite dimensional complex Banach space $X$ such that $M_{T}$ is a closed connected subset of the unit sphere of $X$. Then for $A \in L(X), T \perp_{B} A \Leftrightarrow \exists \theta \in[0, \pi]$ and $x, y \in M_{T}: A x \in(T x)_{\gamma}^{+}$for all $\gamma$ with $\arg \gamma \in[\theta-\pi, \theta]$ and $A y \in(T y)_{\gamma}^{+}$for all $\gamma$ with $\arg \gamma \in[\theta, \theta+\pi]$.

\section{Geometric Properties}

Definition 6.1. [14] Let $x, y \in X$ and $T=\{\mu \in K:|\mu|=1\}$. Then $\mathrm{x}$ is said to be norm parallel to y if $\|x+\mu y\|=\|x\|+\|y\|$ for all $\mu \in T$.

Norm parallelism is symmetric as well as homogeneous; whereas, Birkhoff-James orthogonality is homogeneous but not symmetric in a Banach space. [14] In the case of Hilbert space, two elements are linearly dependent iff they are norm- parallel; however, in normed spaces two linearly dependent vectors are norm-parallel, but the converse may not be true. For instance, $(1,1)$ and $(1,0)$ are norm parallel but not linearly dependent. Depending on the concept of Birkhoff-James orthogonality and strong Birkhoff-James orthogonality Paul et al.[14] introduce a new geometric notion of semi-rotund point. For any normed linear space $\mathrm{X}, \beta \neq x \in X$ is said the semi-rotund point of $\mathrm{X}$ if $\exists y \in X: x \perp_{S B} y$. If for every $x \neq 0 \in X, \mathrm{x}$ is a semi-rotund point, the normed space $\mathrm{X}$ is said to be semi-rotund space. Dragomir introduced the concept of approximate Birkhoff-James orthogonality [15] as follows: $\mathrm{x}$ is said to be approximate Birkhoff-James orthogonal to $\mathrm{y}$ if $\|x+\mu y\| \geq(1-\epsilon)\|x\|$ for all $\mu \in K$ and $\epsilon \in[0,1]$; however, Chmielinski [14, 16] defined approximate Birkhoff-James orthogonality as ; $x \perp_{D}^{\epsilon} \Leftrightarrow\|x+\mu y\| \geq \sqrt{1-\epsilon^{2}}\|x\|$ for all $\mu \in K$. The concept of approximate parallelism was developed by Zamani and Moslehian [17. by stating that $\mathrm{x}$ is approximately parallel to $\mathrm{y}$ if inf $\{\|x+\lambda y\|: \lambda \in K\} \leq \epsilon\|x\|$ for all $\epsilon \in[0,1]$.

Proposition 6.2. 14 let $X$ be a bounded linear operator form a normed space $X$ to normed space $Y$ and $x \in M_{T}$. Then for any $\epsilon \in[0,1]$ and $y \in X$, we have $x\left\|_{y}^{\epsilon} \Rightarrow T x\right\| \|^{\epsilon} T y$.

Theorem 6.3. 14 Let $T$ and $A$ are compact linear operators form a reflexive Banach space $X$ to any normed space $Y$. Then $T\left\|A \Leftrightarrow \exists \quad x \in M_{T} \cap M_{A}: T x\right\| A x$. 
Theorem 6.4. 14 If $T$ and $A$ are bounded linear operators form a normed space $X$ to $Y$. Then $T\left\|A \Leftrightarrow \quad \exists\left\{x_{n}\right\} \in S_{X}: \lim _{n \rightarrow \infty}\right\| T x_{n}\|=\| T\left\|, \lim _{n \rightarrow \infty}\right\| A x_{n}\|=\| A \|$ and $\lim _{n \rightarrow \infty}\left\|T x_{n}+\mu A x_{n}\right\|=\|T\|+\|A\|$, for some $\mu \in K$.

Proposition 6.5. 14 Let $T$ be a bounded linear operator form a normed space $X$ into normed space $Y$ and let $x \in M_{T}$. Then $T x \perp_{D}^{\epsilon} T y \Rightarrow x \perp_{D}^{\epsilon} y$ for any $\epsilon \in[0,1]$ and $y \in X$.

Theorem 6.6. 14 let $T$ and $A$ are bounded linear operators from finite dimensional Banach spaces $X$ to $Y$. Then $T \perp_{S B} A \Leftrightarrow \forall \epsilon>0, \exists \mu_{\epsilon}>0: \forall|\mu|<\mu_{\epsilon}, \exists y_{\mu} \in$ $\left(\cup_{x \in M_{T}} B(x, \epsilon)\right) \cap S_{x}:\left\|T y_{\mu}+\mu A y_{\mu}\right\|>\|T\|$.

Theorem 6.7. 14] Let $T$ and $A$ are compact linear operators fron a reflexive Banach space $X$ to any normed space $Y$ be such that $T \perp_{B} A$ but $T \perp_{S B} A$. Then there exists $x \in M_{T}$ such that $T x \perp_{B} A x$.

Theorem 6.8. 14 Let $T$ and $A$ are bounded linear operators from a normed space $X$ to $Y$. If $T \perp_{B} A$ but $T \perp_{S B} A$, then there exists a sequence $\left\{x_{n}\right\}$ in $S_{X}$ such that $\left\|T x_{n}\right\| \rightarrow\|T\|, A x_{n} \rightarrow 0$ or there exist a sequence $\left\{x_{n}\right\}$ in $S_{X}$ and sequence $\left\{\epsilon_{n}\right\}$ in $\mathbb{R}^{+}$ such that $\left\|T x_{n}\right\| \rightarrow\|T\|, \epsilon_{n} \rightarrow 0$, and $T x_{n} \perp_{D}^{\epsilon_{n}} A x_{n}$.

\section{Relation Between Birkhoff-Jame, Robert, And isosceles orthogonality IN TERMS OF BOUNDED LINEAR OPERATORS}

Recently, Bottazzi et al. in [18] has introduced a new generalization of earlier results on orthogonality of bonded linear operators. They discussed about Birkhoff-James,Isosceles, and Robert orthogonality in Banach spaces in terms of bounded linear operators. For better description of Birkhoff-James orthogonality, they introduced the sets, $\mathscr{O}=\left\{x \in S_{X}: T x \perp_{B} A x\right\}$ for any $T, A \in B(X)$ and $M_{T}=\left\{x \in S_{X}:\|T x\|=\|T\|\right\}$.

For any bounded linear operator $\mathrm{A}$ on the Hilbert space $\mathrm{H} ; A^{*}, R(A)$, and $N(A)$ denotes the adjoint, range and kernal of $\mathrm{A}$ respectively. The bounded linear operators $\mathrm{A}$ and $\mathrm{B}$ in a real or complex Hilbert space $\mathrm{H}$ have a disjoint support if $A B^{*}=B A^{*}=0$.

Theorem 7.1. 18] Let $X$ be reflexive Banach space and $Y$ be Banach spaces, either both real, or both complex. Let $T$ and $A$ are compact linear operators from $X$ to $Y$ be such that for any $x_{0} \in S_{X}$,

$$
M_{T}= \begin{cases} \pm x_{0} & \text { in the real case } \\ e^{i \theta} x_{0}: \theta \in[0,2 \pi] & \text { in the complex case }\end{cases}
$$

Then $T \perp_{B} A \Leftrightarrow \mathscr{O}_{T, A} \cap M_{T} \neq \phi$.

Theorem 7.2. [18] Let $T$ and $A$ are compact linear operators from a reflexive Banach space $X$ to any real Banach space $Y$. If $T$ is Birkhoff-James orthogonal to A, then the set $\mathscr{O}_{T, A}$ is non-empty.

Theorem 7.3. 18 Let $X, Y$ be two Banach spaces, either both real,or, both complex. let and $T$ and $A$ are bounded linear operators from $X$ to $Y$. Then, $\mathscr{O}_{T, A}=S_{X} \Rightarrow T \perp_{B} A$.

Theorem 7.4. 18 A real or complex Hilbert space $H$ is of finite dimensional if and only if for any bounded linear operators in $H, T \perp_{B} A \Rightarrow \mathscr{O}_{T, A} \neq \phi$.

Proposition 7.5. [18] For any bounded linear operators $A$ and $T$ in a real or complex Hilbert space $H$ satisfying $T^{*} A=0$, then the following statements holds:

(i) $A \perp_{B} T$ and $T \perp_{B} A$,

(ii) $A \perp_{R} T$, and in particular, $A \perp_{I} T$

Proposition 7.6. 18 Let $X$ be real or complex normed space. Let $x, y \in X$ and assume that $x+y \perp_{B} y$ and $x-y \perp_{B} y$. Then $x \perp_{I} y$ 
Remark 7.7. In order to illustrate the concept regarding to the converse part of the above proposition Sain et al in [18] introduced strongly Isosceles orthogonality in the real Banach space by stating that: An element $x \in X$ is said to strongly orthogonal to $y \in X$ (written as $x \perp_{S I} y$ ) if the following conditions are satisfied;

(i) $x \perp_{I} y$,

(ii) there exists a real sequence $\left\{\lambda_{n}\right\}_{n \in \mathbb{N}}$, with $\lambda_{n}>0$ such that $\lim _{n \rightarrow \infty} \lambda_{n}=0$ and $x \perp_{I} \lambda_{n} y$ for all $n \in \mathbb{N}$.

Theorem 7.8. 18 Let $x, y \in X$. Then $x \perp_{S I} y \Rightarrow x \perp_{B}^{r} y$ and in particular and is $X$ is real normed space then $x \perp_{S I} y \Rightarrow x \perp_{B} y$.

\section{BIRKHOFF-JAMES ORTHOGONALITY BY APPLYING SEMI-INNER PRODUCT}

The concepts of Birkhoff-James orthogonality has been widely used by various researchers since 1935. The latest research on this topic by Sain, Mal, and Paul [19] have studied Birkhoff-James orthogonality of compact linear operators between Hilbert space and Banach spaces by applying the notion of semi-inner product in normed linear spaces.

Definition 8.1. [19] For any normed linear space $\mathrm{x}$, A scalar valued function (.,.) : $X \times X \rightarrow K$ is a semi-inner product if for any $\xi, \eta \in K$ and for any $x, y, z \in X$, it satisfies the following conditions:

(i) $(\xi x+\eta y, z)=\xi(x, z)+\eta(x, z)$,

(ii) $(x, x)>0$, whenever $x \neq 0$.

(iii) $|(x, y)|^{2} \leq(x, x)(y, y)$,

(iv) $(x, \xi y)=\bar{\xi}(x, y)$.

Every semi-inner product space is a normed space with the norm $\|x\|^{2}=(x, x)$ and the norm of any normed space can be generated through a semi-inner product in infinitely many ways. Sain et al. in [19] characterized the Birkhoff-James orthogonality set of any compact linear operators between a reflexive Banach space any Banach spaces. They also proved that there is an relationship between the concept of semi-inner product spaces and the sets $x^{+}=\{y \in X:\|x+\gamma y\| \geq\|x\|$ for $\gamma \geq 0\}$ and $x^{+}=\{y \in X:\|x+\gamma y\| \geq\|x\|$ for $\gamma \leq 0\}$

Theorem 8.2. [19] Let $T$ and $A$ be compact linear operators from a reflexive Banach space $X$ to any Banach space $Y$. If any one of the following conditions holds;

(i) $M_{T}$ is a connected subset of $S_{X}$.

(ii) $M_{T}$ is not connected but $M_{T}=D \cup(-D)$, where $D$ is a non-empty subset of $S_{X}$. Then $T \perp_{B} A \Leftrightarrow \exists x \in M_{T}: T x \perp_{B} A x$.

Theorem 8.3. [19] For a finite-dimensional Banach space $X$, the following statements are are equivalent.

(i) For any linear operator $T$ on $X, M_{T}$ is the unit sphere of some subspace of $X$.

(ii) For any linear operator $T$ on $X, M_{T}=D_{T} \cup\left(-D_{T}\right)$, where $D_{T}$ is connected subset of $X$.

(iii) $X$ is an Euclidean space.

As an correlation between the semi-inner product space and geometric concepts of the sets Sain et al. proved the following theorem.

Theorem 8.4. [19] Let $x, y \in X$, where $X$ is a normed linear space. Then the following are true.

(i) $y \in x^{+}$iff there exists a semi-inner product $(.,$.$) on X$ with $(y, x) \geq 0$.

(ii) $y \in x^{-}$iff there exists a semi-inner product $(.,$.$) on X$ with $(y, x) \leq 0$. 
Theorem 8.5. Let $T$ and $A$ be compact linear operators from a reflexive Banach space $X$ to any Banach space $Y$ be such that $T \perp_{B} A$. let $\mathscr{O}_{Y}$ denotes the collection of all semi-inner product on $Y$. Then

$$
\|T\|=\left\{\begin{array}{l}
\sup \left\{(T x, y): x \in S_{X}, y \in S_{Y},(., .) \in \mathscr{O}_{Y},(A x, y) \geq 0\right\} \\
\sup \left\{(T x, y): x \in S_{X}, y \in S_{Y},(., .) \in \mathscr{O}_{Y},(A x, y) \leq 0\right\}
\end{array}\right.
$$

Theorem 8.6. [19] Let $T$ and $A$ be bounded linear operators form a normed space $X$ to $Y$ be such that $T \perp_{B} A$. Ley $\mathscr{O}_{Y}$ denotes the collection of semi-inner product space on $Y$. Let $\epsilon>0$ be arbitrary but fixed after $A$ choice. Then

(i) $\|T\|=\max \left\{l_{1}(\epsilon), l_{2}(\epsilon)\right\}=\max \left\{l_{1}(\epsilon), l_{3}(\epsilon)\right\}$, where

(ii) $l_{1}(\epsilon)=\sup \left\{(T x, y): x \in S_{X}, y \in S_{Y},(.,.) \in \mathscr{O}_{Y},|(A x, y)|<\epsilon\right\}$

(iii) $l_{2}(\epsilon)=\sup \left\{(T x, y): x \in S_{X}, y \in S_{Y},(.,.) \in \mathscr{O}_{Y}, A x \in(y)^{+\epsilon}\right\}$

(iv) $l_{3}(\epsilon)=\sup \left\{(T x, y): x \in S_{X}, y \in S_{Y},(.,.) \in \mathscr{O}_{Y}, A x \in(y)^{-\epsilon}\right\}$

Theorem 8.7. [19] Let $X$ be normed linear space such that $X^{*}$ is strictly convex. Let $f, g \in X^{*}$ be such that $f \perp_{B} g$. then

$$
\|f\|=\left\{\begin{array}{l}
\sup \left\{f(x): x \in S_{x}, g(x) \geq 0\right\} \\
\sup \left\{f(x): x \in S_{x}, g(x) \leq 0\right\} .
\end{array}\right.
$$

Theorem 8.8. [19] Let $T$ and $A$ are compact linear operators from a reflexive Banach space $X$ to any Banach space $Y$ be such that for each $\lambda \in \mathbb{R}, M_{T+\lambda A}=D_{\lambda} \cup\left(-D_{\lambda}\right)$, where $D_{\lambda}$ is a non-empty connected subset of $S_{X}$. Let $\mathscr{O}_{Y}$ denotes the collection of all semi-inner product space on $Y$. Then

$$
\operatorname{dist}(T, \operatorname{span}\{A\})=\sup \left\{(T x, y): x \in S_{X}, y \in S_{Y},(., .) \in \mathscr{O}_{Y},(A x, y)=0\right\} .
$$

Theorem 8.9. [19] Let $X$ be a reflexive Banach space and $Y$ be any Banach space. Let $Z$ be a finite dimensional subspace of $K(X, Y)$. Let $T \in K(X, Y) \backslash \mathscr{Z}$. Let us further assume that for any $\lambda \in \mathbb{R}$ and for any $A \in \mathscr{Z}, M_{T+\lambda A}=D_{\lambda, A} \cup\left(-D_{\lambda, A}\right)$, where $D_{\lambda, A}$ is non-empty connected subset of $S_{X}$. Then there exist $A_{0} \in \mathscr{Z}$ such that

$$
\operatorname{dis}(T, \mathscr{Z})=\sup \left\{(T x, y): x \in S_{X}, y \in S_{Y},\left(A_{0} x, y\right)=0\right\} .
$$

Moreover, $A_{0}$ is the best approximation of $T$ in $\mathscr{Z}$.

\section{Modular Birkhoff orthgonality in Banach modules}

We have already mentioned that Rajic et al. in 8 studied Birkhoff-James orthogonality in a Hilbert $C^{*}$-modules over a $C^{*}$-algebra. The most current research as generalization of Birkhoff-James orthogonality from Hilbert space to Banach spaces in [20], Sain and Tanaka studied the stronger version of modular Birkhoff-James orthogonality in the set of bounded and compact linear operators. In order to prove their study they introduced the following notions: $X^{\perp}=\left\{y \in X: x \perp_{B} y\right\}$ and $M_{A}=\left\{x \in S_{X}:\|A x\|=\|A\|\right\}$. An element $x \neq 0 \in X$ is said to be smooth point in $\mathrm{X}$ if $\mathscr{T}(x)=\left\{f \in S_{X}^{*}: f(x)=\|x\|\right\}$ is a singleton set. For any Banach space $\mathrm{X}$, an element $x \in X$ is said to be left symmetric in $\mathrm{X}$ if for any $y \in X, x \perp_{B} y \Rightarrow y \perp_{B} x$. Similarly $\mathrm{x}$ is said to be right symmetric in $\mathrm{X}$ if for any $y \in X, y \perp_{B} x \Rightarrow x \perp_{B} y$. If $\mathrm{x}$ is both left as well as right symmetric, then $\mathrm{x}$ is said to be a symmetric point.

Definition 9.1. 20] A Banach space $\mathrm{X}$ is called a right $\mathscr{A}$-module (where $\mathscr{A}$ is a Banach algebra) if there exists a mapping of $X \times \mathscr{A}$ into $\mathrm{X}$ such that for each $a, b \in \mathscr{A}$ and $x \in X$, $x(a b)=(x a) b$ and $\|a x\| \leq\|x\|\|a\|$.

An element $x \in X$ is said to be right-modular Birkhoff-James orthogonal to $y \in X$, if $x \perp_{B}$ ya for all $a \in \mathscr{A}$ and left-modular Birkhoff-James orthogonal to y if $x \perp_{B}$ ay for all $a \in \mathscr{A}$. 
Theorem 9.2. 20] Let $T$ and $A$ be compact linear operators form a reflexive real Banach space $X$ to any real Banach space $Y$ such that $M_{A}=\left\{ \pm x_{0}\right\}$ for some $x_{0} \in S_{X}$. Then $A_{B(X)}^{\perp} \Leftrightarrow T(X) \subset\left(A x_{0}\right)^{\perp}$.

Definition 9.3. 20 A Banach space $\mathrm{X}$ is said to be Kadets-Klee if whenever $\left\{x_{n}\right\}$ is a sequence in $\mathrm{X}$ and $x \in X$ is such that $\left\{x_{n}\right\}$ converges weekly to $\mathrm{x}$ and $\lim _{n \rightarrow \infty}\left\|x_{n}\right\|=\|x\|$, then $\lim _{n \rightarrow \infty}\left\|x_{n}-x\right\|=0$.

Theorem 9.4. 20] Let $A$ be a compact linear operator from a reflexive Kadets-Klee real Banach space to any real Banach space be such that $M_{T}=\left\{ \pm x_{0}\right\}$ for some $x_{0} \in S_{X}$. Then given any bounded linear operator $T \in B(X, Y), A \perp_{B(X)} T \Leftrightarrow T(X) \subset\left(A x_{0}\right)^{\perp}$.

Theorem 9.5. 20] Let $X, Y$ be real Banach spaces. Let $A \in B(X, Y)$ be a smooth point in $B(X, Y)$ such that $M_{A} \neq 0$. Then given any $T \in B(X, y), A \perp_{B(X)} T \Leftrightarrow T(X) \subset$ $\left(A x_{0}\right)^{\perp}$, where $M_{A}=\left\{ \pm x_{0}\right\}$.

Theorem 9.6. Let $T$ and $A$ are compact linear operators from a reflexive complex $B a-$ nach space $X$ to any complex Banach space $Y$ be such that $M_{A}=\left\{e^{i \theta} x_{0}: \theta \in[0,2 \pi]\right\}$ for some $x_{0} \in S_{X}$. Then given any compact linear operator $T, A \perp_{B(X)} T \Leftrightarrow T(X) \subset$ $\left(A x_{0}\right)^{\perp}$.

Theorem 9.7. 20] Let $T$ and $A$ are compact linear operators from a reflexive real Banach Space $X$ to any real Banach space $Y$ be such that $M_{A}=\left\{ \pm x_{0}\right\}$ for some $x_{0} \in S_{X}$. Then given any compact linear operator $T, A \perp_{B(Y)}^{*} T=\Leftrightarrow T x_{0}=0$. Moreover, if $X$ is KadetsKlee, then same is true for any $T \in B(X, y)$.

Theorem 9.8. [20] Let $T$ and $A$ are compact linear operators from a reflexive complex Banach space to any complex Banach space $Y$ be such that $M_{A}=\left\{e^{i \theta} x_{0}: \theta \in[0,2 \pi]\right\}$ for some $x_{0} \in S_{X}$. Then given any $T \in K(X, Y), A \perp_{B(Y)}^{*} T \Leftrightarrow T x_{0}=0$.

If $\mathrm{A}$ is a bounded linear operator from a normed spaces $\mathrm{X}$ to $\mathrm{Y}$, then its adjoint $A^{*} \in B\left(Y^{*}, X^{*}\right)$ is defined by $\left(A^{*} y^{*}\right)=y^{*} A x$ for each $x \in X, y^{*} \in Y^{*}$ and $\left\|A^{*}\right\|=\|x\|$. For any subsets $\mathrm{R}$ and $\mathrm{S}$ of a Banach space $\mathrm{X}, R \perp_{B} S$ if $x \perp_{B} y$ for all $x \in R$ and $y \in S$.

Proposition 9.9. 20] Let $T$ and $A$ are bounded linear operators from a Banach space $X$ to $Y$. If $A(x) \perp_{B} T(X)$, then $A \perp_{B} T$.

Theorem 9.10. [20] Let $X$ and $Y$ be finite dimensional Banach spaces with $\operatorname{dim}(X) \geq$ $\operatorname{dim}(Y)>0$, and let $A \in B(X, y)$ and suppose that $A(X)=Y$. Then $A$ is right symmetric for $\perp_{B(X)}$ in $B(X, Y)$.

\section{Open PROBLEMS}

Definition 10.1. 21] In a normed linear space $X$,

$$
x \perp y \Leftrightarrow \sum_{k=1}^{m} a_{k}\left\|b_{k} x+c_{k} y\right\|^{2}=0,
$$

where $m \geq 2$ and $a_{k}, b_{k}, c_{k}$ are real numbers such that

$$
\sum_{k=1}^{m} a_{k} b_{k} c_{k}=1, \quad \sum_{k=1}^{m} a_{k} b_{k}^{2}=\sum_{k=1}^{m} a_{k} c_{k}^{2}=0
$$

Problem 10.2. Birkhoff-James, Robert, and isosceles orthogonality has been studied in terms of linear operators in Hilbert space and general Banach spaces. This fact raises a question- can Carlsson orthogonality(in particular Pythagorean orthogonality) be characterized in terms of operators in Hilbert $C^{*}$ as well as Banach modules? 
Problem 10.3. According to proposition-7.8 in 20 if two bounded linear operators in a real or complex Hilbert space satisfy $T^{*} A=0$, then these operators are Birkhoff-James, Robert and isosceles orthogonal. This fact leaves behind a question if we can prove the condition of Pythagorean orthogonality by introducing some different nature of operators $\mathrm{A}$ and $\mathrm{T}$ in the same space or not.

\section{Acknowledgment}

We are thankful to Nick Simon's Foundation and Nepal Mathematical Society for the partial support of our research. We also would like to express deep gratitude towards reviewers and the Editor in Chief of the journal for their valuable suggestions and feedback to improve our manuscript.

\section{REFERENCES}

[1] G. Birkhoff, Orthogonality in linear metric spaces, Duke Math. J. Vol 1(1935)pp. 169-172.

[2] J. Sikoraska, Orthogonalities and functional equations, Aquat. Math. 89(2015),215-277.

[3] R. C. James, Orthogonality and linear functional equations in normed linear spaces, trans AMS, vol.61, no.2(1947)pp265,292.

[4] R. Bhatia, P. Semrl, rthogonality of matrices and some distance problems, Linear algebra and its applications 287(1999), 77-85.

[5] C. Benitz, M. Fernandez, M.L. Soriano, Orthogonality of matrices, linear algebra and its applications, 422(2007), 155-163.

[6] C. Zhi-Zhi, L. Wei, L. Lulin, Projections Birkhoff orthogonality and angles in normed spaces, Communications in Mathematical research, 27(4), 2011, 378-384.

[7] A. Askarizades, M. Dehghan, G-frames as a special frames, Turk. J. Math, 37(2013)60-70.

[8] L. Arambasic, R. Rajic, The Birkhoff-James orthogonality in Hilbert $C^{*}$-modules, Linear algebra and its applications, 437(2012) 1913-1929.

[9] D. Sain, K. Paul, Operator norm attainment and inner-product spaces, linear algebra and its applications, 439(2013) 2448-2452.

[10] D. Sain. K. Paul, S. Hait, Operator norm attainment and Birkhoff-James orthogonality, Linear algebra and its applications, 476(2015)85-97.

[11] K. Paul, D. Sain, K. Jha, On strong orthogonality and strictly convex normed linear space, Journal of inequalities and applications, 2013, no.242.

[12] K. Paul, D. Sain, A. Mall, K. Mandal, Orthogonality of bounded linear operators on complete Banach space, Adv.Oper. Theory, 3(2018), no. 3, 699-709.

[13] D. Sain, Birkhoff-James orthogonality of linear operators on finite dimensional Banach spaces, J. Math. Anal. Appl. 447(2017), no. 2, 860-866,

[14] A. Mal, D. Sain, K. Paul, On some geometric properties of operator spaces, Banach J. Math. Anal. 13(2019), no. 1, 174-191.

[15] S. S. Dragomir, On approximation of continuous linear functional in normed linear spaces, An.Univ. Timisoara. Stiint. Math, 29(1991), no.1, 51-58.

[16] Chmielinski, Linear mappings approximately preserving orthogonality, J. Math. Anal. Appl. 304(2005), no. 1, 158-169.

[17] A. Zamani, M.S. Moslehian, Norm parallelism in the geometry of Hilbert $C^{*}$-modules, Indag. Math.(N.S), 27(2016), no. 1, 266-281.

[18] T. Bottazzi, C. Conde, D. Sain, A study of orthogonality of bounded linear operators, Banach. J. Math. Anal,(2020).

[19] D. Sain, A. Mal and K. Paul, Some remarks on Birkhoff- James orthogonality of linear operators, Expositiones Mathematicae (2019),https : //doi.org/10.1016/j.exmath.2019.01.001.

[20] D. Sain, R. Tanaka, Modular Birkhoff-James orthogonality in $B(X, Y)$ and $K(X, Y),(2020)$ Banach J. Math. Anal. https://doi.org/10.1007/s43037-020-00064-z.

[21] S. O. Carlsson, Orthogonality in normed linear spaces, Ark. Mat. 4 (1962), 297-318 (1962).

Bhuwan Prasad Ojha: bhuwanp.ojha@apexcollege.edu.np

PhD Scholar, Central Department of Mathematics, Tribhuvan University, Kathmandu, Nepal

Prakash Muni Bajracharya: pmbajracharya13@gmail.com

Department of Mathematics, Tribhuvan University, Kathmandu, Nepal 